\title{
Derivate von Aminosäuren.
}

\author{
II. Mitteilung.
}

2. Verbindungen mit Fetteäuren.

Von

Emil Abderhalden und Casimir Funk.

(Aus dem physiologischen Institut der tierärztlichen Hochschule, Berlin.)

(Der Redaktion zugegangen am 26. Januar 1910.)

Bei der Darstellung von Verbindungen, die aus Aminosäuren und Fettsäuren bestehen, bedienten wir uns der gleichen Methoden, wie sie von Emil Fischer für die Bildung von Polypeptiden angewandt worden sind; wir verwendeten nur an Stelle der Halogenacylchloride die halogenfreien Fettsäurechloride. Denselben Weg haben Bondi ${ }^{1}$ ) und seine Mitarbeiter zur Darstellung derartiger Produkte eingeschlagen. Es erfolgt Kuppelung des Carboxyls der Fettsäure mit der Aminogruppe der Aminosäure:

$\mathrm{CH}_{2} \mathrm{R} \cdot \mathrm{CO} \mathrm{Cl}+\mathrm{H} \mathrm{NH} \cdot \mathrm{CH}_{2} \cdot \mathrm{COOH}=\mathrm{CH}_{2} \mathrm{R} \cdot \mathrm{CO} \cdot \mathrm{NH} \cdot \mathrm{CH}_{8} \cdot \mathrm{COOH}$.

Derartige Verbindungen sind von Bondi als Lipopeptide bezeichnet worden. Sie sollten ihm als Ausgangspunkt zu Studien über Fett-Eiweißverbindungen dienen. Es ist fraglich, ob aup diesem Wege ein Einblick in das Verhalten von Kombinationen von Fett- und Eiweißstoffen resp. von Verbindungen zwischen Bausteinen dieser Körperklasse gewonnen werden

1) S. Bondi, Über Lipoproteide und die Deutung der degenerativen Zellverfettung. II. Lipopeptide, ihre Bedeutung, Synthese und Eigenschaften (Lauryl-glycin und Laurylalanin). Biochem. Zeitschr., Bd. XVII, S. 543, 1909. S. Bondi und Th. Frankl: III. Synthese von Palmitylglycin und Palmitylalanin. Ebenda, Bd. XVII, S. 553, 1909, und IV. Über das Verhalten von Lipopeptiden gegenüber Fetten. Ebenda, S. 555. 
kann. Die von Bondi und, wie unten mitgeteilt, auch von uns dargestellten Verbindungen haben nicht, wie der Name Lipopeptid vermuten lassen könnte, direkte Beziehungen $\mathrm{zu}$ den Polypeptiden. Wir haben vielmehr Verbindungen vor uns, die in einem wesentlichen Punkt sich von der genannten Gruppe von Verbindungen unterscheiden. Sie besitzen nämlich im Gegensatz zu den Polypeptiden keine freie Aminogruppe. Um Mißverständnissen vorzubeugen, wäre es wünschenswert, den Namen Lipopeptid ganz fallen zu lassen oder ihn für Verbindungen zwischen Fettsäuren und Aminosäuren zu verwenden, welche eine freie Aminogruppe besitzen. Unsere Absicht war ursprünglich, Verbindungen vom letzteren Typus darzustellen. Alle unsere Bemühungen nach dieser Richtung waren bisher ohne Erfolg. Bei dieser Gelegenheit hatten wir eine Anzahl von Kuppelungen von Fettsäurechloriden mit Aminosäuren ausgeführt und die entstandenen Verbindungen genauer untersucht. Wir teilen die gefundenen Ergebnisse im Anschlusse an die analogen Untersuchungen von Bondi und seinen Mitarbeitern mit. Bemerkt sei noch, daß es sehr schwierig ist, speziell die Verbindungen von Fettsäuren und Tyrosin in völlig reinem Zustande zu erhalten. Bei der totalen Hydrolyse von "analysenreinen» Substanzen mit sehr gut stimmenden Analysenzahlen fanden wir wiederholt zu hohe Werte für Tyrosin. Um festzustellen, ob derartige Kombinationen von Fettsäuren und Aminosäuren im Organismus gespalten werden, haben wir Tyrosinfettsäureverbindungen an einen Alkaptonuriker verfüttert und das Verhalten der Homogentisinsäure verfolgt. Über das Resultat dieser Untersuchung soll später berichtet werden.

$$
\begin{gathered}
\text { Palmityl-glycin. } \\
\mathrm{CH}_{3}\left(\mathrm{CH}_{2}\right)_{14} \mathrm{CO} \cdot \mathrm{NH} \cdot \mathrm{CH}_{2} \cdot \mathrm{COOH} \text {. }
\end{gathered}
$$

1. Darstellung aus Glykokoll und Palmitinchlorid:

$5 \mathrm{~g}$ Glykokoll wurden in $66 \mathrm{ccm}$ n-Natronlauge gelöst und abwechselnd $15 \mathrm{~g}$ Palmitinchlorid und $66 \mathrm{ccm}$ n-Natronlauge in kleinen Portionen unter lebhaftem Umschütteln zugefügt. Nach Beendigung der Reaktion wurde von ausgeschiedenen 
Produkten abfiltriert, das Filtrat ausgeäthert und die wässerige, alkalische Lösung nach Abtrennung des Äthers abfiltriert. Nach dem Ansäuern mit n-Salzsäure schied sich ein flockiger Niederschlag ab. Er wurde abgesaugt, mit wenig Alkohol und Äther gewaschen und aus heißem Alkohol umkrystallisiert. Beim Abkühlen schied sich Palmityl-glycin in Form von zu Büscheln vereinigten feinen Nadeln ab. Ausbeute an Rohprodukt $15 \mathrm{~g}$, an reinem Produkt $10 \mathrm{~g}$. Sintert bei $119^{\circ}$, schmilzt bei $123^{\circ}$ (unkorr.) $=125^{\circ}$ (korr.). Unlöslich in Wasser, ziemlich schwer in kaltem, leichter in heißem Alkohol löslich, unlöslich in Äther.

$$
\begin{aligned}
& 0,1496 \mathrm{~g} \text { Substanz gaben } 0,3789 \mathrm{~g} \mathrm{CO}_{2} \text { und } 0,1488 \mathrm{~g} \mathrm{H}_{2} \mathrm{O} \text {. } \\
& 0,1549
\end{aligned}
$$

Berechnet für $\mathrm{C}_{18} \mathrm{H}_{35} \mathrm{O}_{3} \mathrm{~N}(313): 69,01 \% \mathrm{C}, 11,18 \% \mathrm{H}, 4,49 \% \mathrm{~N}$.

Gefunden: $69,08 \% \mathrm{C}, 11,05 \% \mathrm{H}, 4,47 \% \mathrm{~N}$.

2. Darstellung aus Glykokolläthylester und Palmitinchlorid:

Palmityl-glycinäthylester $\mathrm{CH}_{3}\left(\mathrm{CH}_{2}\right)_{14} \mathrm{CO}-\mathrm{NH}-\mathrm{CH}_{2}-\mathrm{COOC}_{2} \mathrm{H}_{5}$.

$1,5 \mathrm{~g}$ Glykokollester, gelöst in $40 \mathrm{ccm}$ trockenen Chloroforms, wurden mit 2,8 g Palmitinchlorid geschüttelt. Das Chloroform wurde abgedunstet, das Reaktionsprodukt mit Wasser gewaschen und aus Alkohol umkrystallisiert. Ausbeute 1,2 g. Nädelchen. Schmelzpunkt 80-85 ${ }^{\circ}$. Löslich in Alkohol, unlöslich in Äther.

$0,2472 \mathrm{~g}$ Substanz verbrauchten $7,9 \mathrm{ccm} \mathrm{n} / \mathbf{1 0}_{-}-\mathrm{H}_{8} \mathrm{SO}_{4}$.

Berechnet für $\mathrm{C}_{80} \mathrm{H}_{39} \mathrm{O}_{8} \mathrm{~N}=341: 4,1 \% \mathrm{~N}$.

Gefunden: $\quad 4,47 \% \mathrm{~N}$.

$0,8 \mathrm{~g}$ der Substanz wurden mit $20 \mathrm{ccm} \mathrm{n}$-Natronlauge durch Erwärmen verseift. Es findet keine vollständige Lösung statt, ein geringer Teil bleibt ungelöst. Das Filtrat, eine seifenartige Flüssigkeit, wurde mit Salzsäure angesäuert. Das ausgeschiedene Produkt wurde abfiltriert und mit Alkohol und Äther gewaschen. Sintert bei 118-119 ${ }^{\circ}$; klare Schmelze bei 123-124 ${ }^{\circ}$. Mit nach der ersten Art gewonnenem Palmitylglycin gemischt, bleibt der Schmelzpunkt unverändert.

$0,2466 \mathrm{~g}$ Substanz brauchten $8,05 \mathrm{ccm} \mathrm{n} / \mathbf{1 0}-\mathrm{H}_{2} \mathrm{SO}_{4}$.

Berechnet: $4,49 \% \mathrm{~N}$.

Gefunden: $4,55 \% \mathrm{~N}$. 
Palmityl-d-alanin.

$$
\mathrm{CH}_{3}\left(\mathrm{CH}_{2}\right)_{14} \mathrm{CO} \cdot \mathrm{NH} \cdot \mathrm{CH}\left(\mathrm{CH}_{3}\right) \mathrm{COOH} \text {. }
$$

$3,2 \mathrm{~g} \mathrm{~d}$-Alanin, gelöst in $36 \mathrm{ccm} \mathrm{n}$-Natronlauge, und $10 \mathrm{~g}$ Palmitinchlorid und 36,4 ccm n-Natronlauge. Die Lösung wurde mit Salzsäure angesäuert, das ausfallende Produkt abgenutscht (Ausbeute $12 \mathrm{~g}$ ) und aus absolutem Alkohol umkrystallisiert. Ausbeute an reiner Substanz 7,3 g. Nadeln. Sintert bei 1050, schmilzt bei $110^{\circ}$. In kaltem Alkohol schwer, in heißem leicht löslich. In Äther schwer löslich. Leicht löslich in heißem Aceton und in Chloroform.

0,1690 g Substanz gaben 0,405̃ $\mathrm{g} \mathrm{CO}_{2}$ und $0,1623 \mathrm{~g} \mathrm{H}_{2} \mathrm{O}$.

$0,2902 \gg>$ verbrauchten $9,2 \mathrm{ccm} \mathrm{n} / 10-\mathrm{H}_{2} \mathrm{SO}_{4}$.

Berechnet für $\mathrm{C}_{19} \mathrm{H}_{37} \mathrm{O}_{3} \mathrm{~N}$ (327): $69,72 \% \mathrm{C}, 11,31 \% \mathrm{H}, 4,28 \% \mathrm{~N}$. Gefunden: $69,13 \% \mathrm{C}, 11,27 \% \mathrm{H}, 4,43 \% \mathrm{~N}$.

$0,2090 \mathrm{~g}$ Substanz drehten in 1,82\% iger absolut alkoholischer Lösung im $2 \mathrm{dm}-$ Rohr $-0,17 \mathrm{~g}$ (Gesamtgewicht der Lösung $11,4290 \mathrm{~g}$, spez. Gew. 0,78$)[\alpha]_{20^{\circ}}^{\mathrm{D}}=-5,98^{\circ}$.

Mono-palmityl-1-tyrosin.

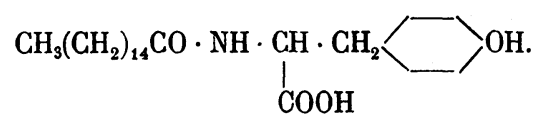

$4,9 \mathrm{~g}$ Tyrosinesterchlorhydrat wurden mit $20 \mathrm{ccm}$ n-Natronlauge versetzt und der freie Ester mit Chloroform extrahiert. Die Lösung wurde über Natriumsulfat getrocknet und mit $5,2 \mathrm{~g}$ Palmitinchlorid gekuppelt. Während der Reaktion schied sich etwas Tyrosinesterchlorhydrat ab, das abfiltriert wurde. Das Filtrat davon wurde im Vakuum zur Trockne eingedampft, der Rückstand, ein Öl, erstarrte bald darauf. Das Produkt wurde nun zur Entfernung von Tyrosinesterchlorhydrat mit Wasser, dann mit $n$-Natronlauge in einer Reibschale zerrieben. Die erhaltene Lösung erwärmten wir, filtrierten dann und säuerten das Filtrat mit Salzsäure an. Rohprodukt 7 g. Es wurde zweimal aus Alkohol umkrystallisiert. Ausbeute an reiner Substanz 3,7 g. Blättchen. Sintert bei $120^{\circ}$, schmilzt bei $133^{\circ}$. In kaltem Alkohol schwer löslich, in warmem leicht löslich, in Äther und Chloroform unlöslich, dagegen in Aceton leicht löslich. Millon positiv. 
$0,1324 \mathrm{~g}$ Substanz lieferten $0,3435 \mathrm{~g} \mathrm{CO}_{2}$ und $0,1197 \mathrm{~g} \mathrm{H}_{\mathbf{2}} \mathrm{O}$.

$0,2042, \quad$ verbrauchten $5,9 \mathrm{ccm} \mathrm{n/10-}-\mathrm{H}_{2} \mathrm{SO}_{4}$.

Berechnet für $\mathrm{C}_{25} \mathrm{H}_{41} \mathrm{O}_{4} \mathrm{~N}(419): 71,59 \% \mathrm{C}, 9,76 \% \mathrm{H}, 3,34 \% \mathrm{~N}$.

Gefunden: $\quad 70,75 \% \mathrm{C}, 10,04 \% \mathrm{H}, 3,24 \% \mathrm{~N}$.

$0,2201 \mathrm{~g}$ Substanz drehten in 1,79\% \% iger absolut alkoholischer Lösung bei Natriumlicht im $2 \mathrm{dm}-\mathrm{Rohr}+0,68$. (Gesamtgewicht der Lösung 12,2571, spezifisches Gewicht 0,78.) $[\alpha]_{\mathrm{D}}^{20^{\circ}}=+24,35$.

2 g Substanz wurden durch 6 stündiges Kochen mit konzentrierter Salzsäure am Rückflußkühler gespalten. Die ausgeschiedene Palmitinsäure wurde abfiltriert. 1,15 g, Schmelzpunkt $62^{\circ}$. Das Filtrat wurde im Vakuum zur Trockene eingeengt. Es verblieben $0,8 \mathrm{~g}$ Tyrosinchlorhydrat. Dasselbe wurde in absolut alkoholischer Lösung mit gasförmiger Salzsäure verestert, die Lösung eingeengt und der Rückstand mit Essigäther ausgekocht. Aus der Lösung schieden sich Nadeln aus, die aus Tyrosinesterchlorhydrat bestanden. $1,02 \mathrm{~g}$, Schmelzpunkt $166^{\circ}$.

Berechnet 1,22 g Palmitinsäure und 1,16 g Tyrosinesterchlorhydrat.

\section{Palmityläther des Palmityl-l-tyrosins.}

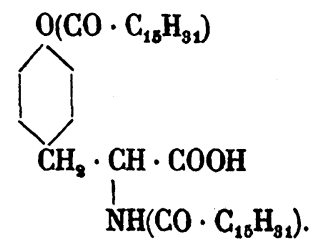

Aus $10 \mathrm{~g}$ l-Tyrosin, in $110,4 \mathrm{ccm} \mathrm{n}$-Natronlauge gelöst, und $15 \mathrm{~g}$ Palmitinchlorid und $54,6 \mathrm{ccm}$ n-Natronlauge gewonnen. Das nach dem Ansäuern ausgefallene Produkt wurde aus absolutem Alkohol umkrystallisiert. Ausbeute $11 \mathrm{~g}$. Nadeln, die keine Rotfärbung mit Millons Reagens geben. Sintert bei $87^{\circ}$, schmilzt bei $95-96^{\circ}$ (unkorr.). In Wasser unlöslich, in kaltem Alkohol schwer, in heißem leichtlöslich (ca. 20\%).

$0,1697 \mathrm{~g}$ Substanz lieferten 0,4626 g CO and 0,1675 $\mathrm{g} \mathrm{H}_{2} \mathrm{O}$.

0,2680 > verbrauchten $4,2 \mathrm{ccm} \mathrm{n} / 10-\mathrm{H}_{8} \mathrm{SO}_{4}$.

Berechnet für $\mathrm{C}_{41} \mathrm{H}_{71} \mathrm{O}_{5} \mathrm{~N}(657): 74,88 \% \mathrm{C}, 10,80 \% \mathrm{H}, 2,13 \% \mathrm{~N}$.

Gefunden: $74,34 \% \mathrm{C}, 10,96 \% \mathrm{H}, 2,19 \% \mathrm{~N}$.

Hoppe-Seyler's Zeitschrift f. physiol. Chemie. LXV. 
$0,2106 \mathrm{~g}$ Substanz in 1,72\% \% iger absolut alkoholischer Lösung drehten bei Natriumlicht im $2 \mathrm{dm}-\mathrm{Rohr}+0,41^{\circ}$. (Gesamtgewicht der Lösung 12,2038, spezifisches Gewicht 0,78.) $[\alpha]_{\mathrm{D}}^{200}=+15,28^{\circ}$.

Palmityläther des Palmityl-3-5-dijod-1-tyrosins.

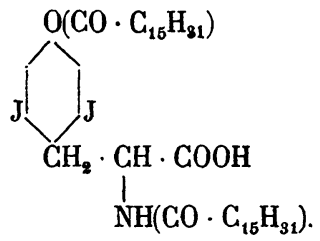

Darstellung wie beim Palmityläther des Palmityl-l-tyrosins. Mikroskopische Nädelchen aus Eisessig, aus Alkohol in nicht definierbaren Krystallaggregaten. Sintert bei $50^{\circ}$, schmilzt bei $55^{\circ}$, klare Schmelze erst bei $62^{\circ}$. Wenig löslich in kaltem Alkohol und Eisessig, Essigäther, Äther, in allen diesen Lösungsmitteln in der Wärme löslich. Sehr wenig löslich in Wasser und Petroläther, wenig löslich in verdünnter Natronlauge und Salzsäure.

$$
\begin{aligned}
& 0,2904 \mathrm{~g} \text { Substanz gaben } 0,1490 \mathrm{~g} \mathrm{AgJ} . \\
& 0,3120 \text { v verbrauchten } 3,9 \mathrm{ccm} \mathrm{n} / 10-\mathrm{H}_{2} \mathrm{SO}_{4} .
\end{aligned}
$$

Berechnet für $\mathrm{C}_{41} \mathrm{H}_{69} \mathrm{O}_{5} \mathrm{NJ}_{2}(909): 1,54 \% \mathrm{~N}, 27,94 \% \mathrm{~J}$. Gefunden: $1,74 \% \mathrm{~N}, 27,5 \% \mathrm{~J}$.

Das optische Verhalten konnte wegen der bei Zimmertemperatur zu geringen Löslichkeit des Präparates nicht bestimmt werden.

$$
\begin{gathered}
\text { Stearyl-glycin. } \\
\mathrm{C}_{17} \mathrm{H}_{85} \cdot \mathrm{CO} \cdot \mathrm{NH} \cdot \mathrm{CH}_{8} \cdot \mathrm{COOH} .
\end{gathered}
$$

Aus 2,4 g Glykokoll, in $32 \mathrm{ccm}$ n-Natronlauge gelöst, durch Kuppeln mit $10 \mathrm{~g}$ Stearinchlorid und $33 \mathrm{ccm}$ n-Natronlauge dargestellt. Die Lösung wurde angesäuert und das ausfallende Produkt 2 mal aus absolutem Alkohol umkrystallisiert. Ausbeute $9 \mathrm{~g}$. Blättchen. Sintert bei $145^{\circ}$, schmilzt bei $155^{\circ}$ (unkorr.). In Wasser unlöslich, in kaltem Alkohol und Äther schwerlöslich, in heißem Alkohol ca. zu 15\% löslich. 
$0,1644 \mathrm{~g}$ Substanz lieferten $0,4211 \mathrm{~g} \mathrm{CO}_{2}$ und $0,1672 \mathrm{~g} \mathrm{H}_{2} \mathrm{O}$.

0,2513 > verbrauchten $7,2 \mathrm{ccm} \mathrm{n} / 10-\mathrm{H}_{2} \mathrm{SO}_{4}$.

Berechnet für $\mathrm{C}_{2 v} \mathrm{H}_{39} \mathrm{O}_{3} \mathrm{~N}(341): 70,38 \% \mathrm{C}, 11,43 \% \mathrm{H}, 4,1 \% \mathrm{~N}$.

Gefunden: $\quad 69,85 \% \mathrm{C}, 11,30 \% \mathrm{H}, 4,01 \% \mathrm{~N}$.

$2 \mathrm{~g}$ Substanz wurden durch 6 stündiges Kochen mit $20 \mathrm{ccm}$ konzentrierter Salzsäure gespalten. Die ausgeschiedene Stearinsäure wurde abfiltriert und getrocknet. 1,62 g. Schmelzpunkt $71^{\circ}$. Das Filtrat wurde im Vakuum eingeengt, mit absolutem Alkohol übergossen und verestert. $0,78 \mathrm{~g}$ Glykokollesterchlorhydrat. Schmelzpunkt $142^{\circ}$. Berechnet $0,44 \mathrm{~g}$ Glykokoll und $1,66 \mathrm{~g}$ Stearinsäure.

$$
\begin{gathered}
\text { Stearyl-d-alanin. } \\
\mathrm{C}_{17} \mathrm{H}_{35} \mathrm{CO} \cdot \mathrm{NH} \cdot \mathrm{CH}\left(\mathrm{CH}_{3}\right) \cdot \mathrm{COOH} .
\end{gathered}
$$

Aus 1,8 g d-Alanin und $6 \mathrm{~g}$ Stearinchlorid. Ausbeute $3,8 \mathrm{~g}$. Nadeln aus heißem Alkohol. Unlöslich in Wasser. Wenig löslich in Äther, Natronlauge, Essigäther, löslich in kaltem Alkohol leicht löslich in heißem Alkohol und Essigäther. Schmelzpunkt $105-108^{\circ}$.

I. 0,1650 g Substanz lieferten $0,4305 \mathrm{~g} \mathrm{CO}_{2}$ und 0,1693 $\mathrm{g} \mathrm{H}_{2} \mathrm{O}$.

II. $0,1800 \gg \quad$ > $5,4 \mathrm{ccm} \mathrm{N}\left(20^{\circ}, 766 \mathrm{~mm}\right)$.

III. 0,4218 》 verbrauchten $10,95 \mathrm{ccm} \mathrm{n} / 10-\mathrm{H}_{2} \mathrm{SO}_{4}$.

Berechnet für $\mathrm{C}_{21} \mathrm{H}_{41} \mathrm{O}_{3} \mathrm{~N}(355): 70,98 \% \mathrm{C}, 11,55 \% \mathrm{H}, 3,94 \% \mathrm{~N}$.

Gefunden: I. $71,16 \%$ C, $11,42 \%$ H, II. $3,52 \%$, III. $3,63 \% \mathrm{~N}$.

0,2924 g Substanz drehten in Alkohol gelöst, im $2 \mathrm{dm}$-Rohr $-0,14^{\circ}$. Gesamtgewicht der Lösung 15,174g. (Spez. Gew. 0,79.) $[\alpha]_{\mathrm{D}}^{200}=-4,55^{0}$.

\section{Stearyläther des Stearyl-1-tyrosins.}

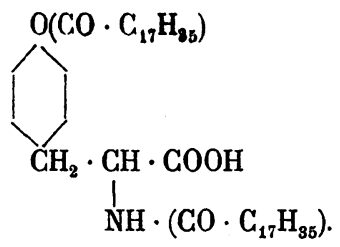

Aus 5,4 g 1-Tyrosin, gelöst in $60 \mathrm{ccm}$ n-Natronlauge, durch Kuppeln mit 18,2 $\mathrm{g}$ Stearinchlorid und $60 \mathrm{ccm}$ n-Natronlauge gewonnen. Das Reaktionsprodukt scheidet sich als dicker, weißer Krystallbrei aus. $2 \mathrm{mal}$ aus Alkohol umkrystallisiert. 
Ausbeute $16 \mathrm{~g}$. Aus Alkohol und Eisessig feine Nadeln. Reaktion mit Millons Reagens negativ. Beginnt bei $88^{\circ} \mathrm{zu}$ sintern, bei $98^{\circ}$ trübe, bei $108^{\circ}$ (unkorr.) klare Schmelze. Unlöslich in Wasser, in Äther und kaltem Alkohol, Eisessig, Methylalkohol wenig löslich, in heißem Alkohol und Eisessig leicht löslich.

$0,1605 \mathrm{~g}$ Substanz lieferten $0,4360 \mathrm{~g} \mathrm{CO}_{2}$ und $0,1642 \mathrm{y}_{2} \mathrm{O}$.

0,3305 > verbrauchten $3,90 \mathrm{ccm} \mathrm{n} / 10-\mathrm{H}_{2} \mathrm{SO}_{4}$.

Berechnet für $\mathrm{C}_{45} \mathrm{H}_{79} \mathrm{O}_{5} \mathrm{~N}(713,6): 73,87 \% \mathrm{C}, 11,07 \% \mathrm{H}, 1,96 \% \mathrm{~N}$.

Gefunden: $74,09 \% \mathrm{C}, 11,44 \% \mathrm{H}, 1,63 \% \mathrm{~N}$.

Wir haben ferner Palmityl-dl-phenylalanin, Palmityl-dlleucin, Stearyl-d-glutaminsäure, Palmityl-cystin, Palmityl-l-tryptophan dargestellt und zur Kuppelung auch einige Chloride der niederen Fettsäuren benutzt. In Rücksicht auf die Untersuchungen von Bondi haben wir unsere Versuche abgebrochen und diese Körper nicht weiter untersucht. Wir werden auf anderem Wege versuchen, Verbindungen mit Fettsäuren zu erhalten, die freie Aminogruppen besitzen. 